\title{
EFECTO DEL CONSUMO DE POLYMNIA SONCHIFOLIA (YACÓN) EN LOS NIVELES DE LÍPIDOS EN PACIENTES DISLIPIDÉMICOS DEL CENTRO GERIÁTRICO DEL HOSPITAL NAVAL, JULIO-NOVIEMBRE DEL 2005
}

\author{
María COLLANTES COSSío 1
}

\begin{abstract}
RESUMEN
Objetivo: El objetivo fue determinar el efecto de la Polymnia Sonchifolia sobre los niveles séricos de colesterol y triglicéridos plasmáticos en pacientes dislipidémicos. Metodología: Estudio tipo prospectivo preexperimental y analítico. La muestra, no aleatoria, estuvo conformada por 50 pacientes. El grupo control estuvo integrado por 18 pacientes mientras que el grupo de tratamiento por 32 pacientes. Resultados: El consumo de yacón redujo significativamente ( $<<0.05$ ) los niveles promedio de VLDL (de 38.1 a $30.2 \mathrm{mg} / \mathrm{dl}$ ), LDL (de 184.1 a $153.6 \mathrm{mg} / \mathrm{dl}$ ), colesterol total (de 267 a $230.1 \mathrm{mg} / \mathrm{dl}$ ) y triglicéridos (de $185.2149 .4 \mathrm{mg} / \mathrm{dl}$ ), sin modificar los niveles de HDL colesterol los cuales se mantuvieron similares hasta el final del estudio. En conjunto, el impacto sobre las variables mencionadas disminuyó significativamente $(\mathrm{p}<0.05)$ el riesgo cardíaco (de 6.0 a 5.0). Conclusiones: El consumo diario de yacón ha mostrado reducir significativamente $(\mathrm{p}<0.05)$ los niveles promedio de VLDL, LDL, colesterol total y triglicéridos sin modificar los niveles de HDL colesterol
\end{abstract}

Palabras clave: Colesterol total, colesterol LDL, colesterol HDL, triglicéridos, dislipidemia, hiperlipemia, hipolipemiante, Polymnia Sonchifolia, fructooligosacáridos, fibra soluble.

\begin{abstract}
Objective: The objective was to determine the effect of Smallanthus Sonchifolius on serum cholesterol and plasma triglycerides in dyslipidemic patients. Methodology: This is a prospective, pre-experimental and analytical study. The non random sample consisted of 50 patients. The control group consisted of 18 patients while the treatment group consisted of 32 patients. Results: The yacon consumption significantly reduced $(p<0.05)$ mean levels of VLDL (from 38.1 to $30.2 \mathrm{mg} / \mathrm{dl}$ ), LDL (from 184.1 to $153.6 \mathrm{mg} / \mathrm{dl}$ ), total cholesterol (from 267 to $230.1 \mathrm{mg} / \mathrm{dl}$ ) and triglycerides (149.4 to $185.2 \mathrm{mg} / \mathrm{dl}$ ) without changing HDL cholesterol levels were maintained similar to the end of the study. Overall, the impact on these variables decreased significantly $(\mathrm{p}<0.05)$ cardiac risk (6.0 to 5.0). Conclusions: Daily consumption of yacon has been shown to reduce significantly $(p<0.05)$ mean levels of VLDL, LDL, total cholesterol and triglycerides without affecting HDL cholesterol levels.
\end{abstract}

Key Words: total cholesterol, LDL cholesterol, HDL cholesterol, triglycerides, dyslipidemia, hyperlipidemia, lipid lowering, smallanthus sonchifolius, fructooligosaccharides, soluble fiber.

${ }^{1}$ Directora E.A.P. Nutrición Humana de la Facultad de Ciencias de la Salud, Universidad Peruana Unión. Docente Asociada. 


\section{INTRODUCCIÓN}

Para el 2001, las enfermedades cardiovasculares (ECVs) representaban la primera causa de muerte en mayores de 65 años en los Estados Unidos de América y eran las responsables de uno de cada tres fallecidos en la Unión Europea ${ }^{1}$. Para el año 2020 se calcula que las muertes por ECVs se incrementarán en un $29 \%$ en mujeres y un $48 \%$ en varones en los países ricos, mientras que en los países en desarrollo, en un lapso de 30 años, las muertes por cardiopatías habrán aumentado un $120 \%$ en mujeres y un $13 \%$ en varones ${ }^{2}$. En los países en desarrollo su incidencia aumenta, lamentablemente, a medida que mejora la situación económica y el nivel de bienestar ( $80 \%$ de los 17 millones de muertes anuales por ECVs ocurren en países pobres o en desarrollo). El urbanismo, la tendencia a tener un estilo de vida más sedentario marcado por el estrés y el terreno ganado por el consumo de carbohidratos refinados, exceso de grasa, bebidas alcohólicas y cigarrillos en detrimento de hábitos de vida más saludables están generando un incremento asociado de la prevalencia de enfermedades como diabetes, obesidad e hipertensión, todas ellas factores de riesgo para desarrollar ECVs.

Es conocido que existe una correlación entre ECVs y altos niveles de lípidos sanguíneos. Los trastornos lipídicos pueden incrementar el riesgo de cardiopatía isquémica en hombres y en mujeres, probablemente por su asociación con otros factores de riesgo como la diabetes mellitus tipo 2, obesidad y la hipertensión $\operatorname{arterial}^{(3)}$. El índice de Castelli o índice aterogénico (Colesterol total / HDL total), por ejemplo, nos muestra si los niveles de HDL son suficientes para "manejar" la carga total de colesterol. (Tabla 1$)^{(4)}$.

\begin{tabular}{|c|c|c|}
\hline Hombre & Mujer & Riesgo coronario \\
\hline$<3,5$ & $<3,4$ & Mitad del promedio \\
\hline $3,5-5,0$ & $3,4-4,5$ & Promedio \\
\hline $5,1-9,6$ & $4,5-7,1$ & Dos veces el promedio \\
\hline $9,7-24$ & $7,2-11$ & Tres veces el promedio \\
\hline
\end{tabular}

Tabla 1. Riesgo de enfermedad coronaria asociado con el índice de Castelli. (Quesada, A. Principales pruebas de Bioquímica Clínica y de Laboratorio, 2003).

En Perú, la Encuesta Nacional de Indicadores Nutricionales, Bioquímicos, Socioeconómicos y Culturales relacionados con las Enfermedades Crónico Degenerativas (2006), mostró que el sobrepeso afectaba al $35.3 \%$ de la población peruana $(39.1 \%$ en los mayores de 60 años); mientras que la obesidad, al $16.5 \%$ de la población ( $16.8 \%$ en los mayores de 60 años). Con respecto a la prevalencia de hipertrigliceridemias y contenido elevado de LDL, mostró que afectaba aproximadamente al $15 \%$ de la población. La prevalencia de hipertrigliceridemia se incrementaba con la edad, siendo los más afectados los pobladores mayores de 50 años. La prevalencia de hipercolesterolemia y prevalencia de LDL era mayor en mujeres (22\%). Mostró además una baja prevalencia de niveles anormales de colesterol HDL ( $<35 \mathrm{mg} / \mathrm{dL})$ en la población evaluada (prevalencia igual al $1 \%)^{(5)}$.

La evidencia científica de los últimos 15 años ha demostrado que los fructooligosacáridos (FOS) -un tipo de fibra con propiedades prebióticas- pueden tener influencia beneficiosa para la salud. Así se ha reportado que reducen el nivel de lípidos en la sangre ${ }^{6,7}$ y el riesgo de cáncer de colon, entre otros ${ }^{8}$. El yacón (Polymnia Sonchifolia) raíz andina y poco conocida aún, perteneciente al grupo de plantas denominadas como raíces y tubérculos andinos (RTA), tiene un alto contenido de inulina y fructooligosacáridos (FOS). La mayoría de las propiedades que se asignan al yacón han sido identificadas de manera indirecta. Casi toda la evidencia proviene de estudios realizados con FOS purificados a partir de la achicoria una planta emparentada con el yacón por contener inulina. Nutricionalmente el yacón posee un valor calórico bajo por su alto contenido de agua y carbohidratos $(12,9 \%)$ (Tabla No 2) $)^{(9)}$. Presenta un alto contenido de fructooligosacáridos entre 60 a $70 \%$ en base seca (Tabla 3) y una baja proporción de azúcares reductores como glucosa, fructosa y sucrosa ${ }^{10,11}$. Generalmente se consume fresco. El tubérculo es dulce y es cortado y añadido a las ensaladas, impartiendo sabor y textura. También se consume sancochado u horneado; en la cocción permanece dulce y ligeramente tostado. A pesar de sus cualidades sólo el $1 \%$ de la población lo consume, ya que este producto llega en forma limitada al mercado. En los Andes frecuentemente se rayan y se exprimen para ser filtrados por una tela para obtener una bebida dulce y refrescante. Algunas veces cuando está concentrado, forma bloques de azúcar, turrón oscuro llamado chancaca. La cáscara puede tener un sabor no muy agradable, por el cual los tubérculos se pelan antes de comerlos ${ }^{(12,13)}$. 


\begin{tabular}{lc}
\hline \multicolumn{1}{c}{ Nutrientes } & Contenido \\
Energía & $54 \mathrm{kcal}$. \\
Fibra & $0.5 \mathrm{mg}$ \\
Ceniza & $0.3 \mathrm{mg}$ \\
Azúcares & $9-13 \mathrm{~g}$ \\
Proteínas & $0.3-0.5 \mathrm{~g}$ \\
Lípidos & $0.01-0.05 \mathrm{~g}$ \\
Ceniza & $0.3 \mathrm{mg}$ \\
Calcio & $23 \mathrm{mg}$ \\
Fósforo & $21 \mathrm{mg}$ \\
Retinol & $12 \mathrm{mcg}$ \\
Tiamina & $0.02 \mathrm{mg}$ \\
Riboflavina & $0.11 \mathrm{mg}$ \\
Niacina & $0.34 \mathrm{mg}$ \\
Ácido ascórbico & $13.1 \mathrm{mg}$ \\
Agua & $86-90 \mathrm{ml}$
\end{tabular}

Tabla 2. Composición química nutricional del yacón (Polymnia Sonchifolia) por $100 \mathrm{~g}$ de porción comestible (Collazos et al, 1975)

\begin{tabular}{|lc|} 
Carbohidrato & Porcentaje \\
\hline FOS & 70 \\
Sacarosa & 15 \\
Fructosa & 10 \\
Glucosa & 5 \\
TOTAL & 100 \\
\hline
\end{tabular}

Tabla 3. Composición relativa de los azúcares del yacón (Fuente: Cisneros-Zevallos, 2002)

El objetivo del presente trabajo fue determinar el efecto de la Polymnia Sonchifolia sobre los niveles séricos de colesterol y triglicéridos plasmáticos en pacientes dislipidémicos.

\section{MATERIAL Y MÉTODOS}

El estudio es de tipo prospectivo preexperimental y analítico. La población de estudio estuvo conformada por pacientes dislipidémicos atendidos en el Centro Geriátrico del Hospital Naval. Luego de haber obtenido la autorización de parte del director de Capacitación, se seleccionaron pacientes con hipercolesterolemia ( $>200 \mathrm{mg} / \mathrm{dl} \mathrm{y}<300 \mathrm{mg} / \mathrm{dl}$ ) e hipertrigliceridemia (> $140 \mathrm{mg} / \mathrm{dl}$ y $<400 \mathrm{mg} / \mathrm{dl}$ ) de ambos sexos con edades entre 35 y 75 años. Se eligieron estos rangos porque valores mayores de colesterol $(>300 \mathrm{mg} / \mathrm{dl})$ y de triglicéridos (>400 mg/dl) implicaban riesgo y tratamiento medicamentoso. La dislipidemia presentada por los pacientes fue la tipo primario (es decir, por defectos propios del metabolismo lipídico y no secundario a una enfermedad) y fue categorizada usando la clasificación primaria en hipercolesterolemia (colesterol plasmático $>200 \mathrm{mg} / \mathrm{dl} \mathrm{y}$ triglicéridos $<200 \mathrm{mg} / \mathrm{dl}$ ), hipertrigliceridemia (triglicéridos $>200 \mathrm{mg} / \mathrm{dl}$ y colesterol $<200$ $\mathrm{mg} / \mathrm{dl}$ ) o hiperlipemia combinada (colesterol y triglicéridos $>200 \mathrm{mg} / \mathrm{dl}$ ) (14). La muestra, no aleatoria, estuvo conformada por 50 pacientes que aceptaron voluntariamente previa explicación del tratamiento y evaluación de los criterios de inclusión. El grupo control estuvo conformado por 18 pacientes, mientras que el grupo de tratamiento por 32 pacientes.

Para el despistaje de las enfermedades excluyentes del estudio, se realizó una anamnesis de los antecedentes patológicos personales.

Se tomó muestra de sangre en ayunas de 12 horas para colesterol total, triglicéridos y HDLc en dos oportunidades al primer día (inicio) y a los 75 días (final del tratamiento). Tanto el colesterol como los triglicéridos fueron analizados por métodos enzimáticos. De la misma forma se registró el peso y talla de los pacientes para determinar su diagnóstico nutricional por medio del índice de masa corporal- IMC al primer día (inicio) y a los 75 días (final) del estudio. Cabe resaltar que no se evaluó dieta por no interferir con el efecto específica del tratamiento con yacón. Los pacientes elegidos no se encontraron en tratamiento dietético (hipocalórico o hipograso). Se indicó a los pacientes del grupo en tratamiento el consumo diario de una unidad de yacón $(120 \mathrm{~g})$ al natural, previamente lavada, pelada o en forma de extracto, de preferencia en ayunas. Se puso énfasis en no consumirla en forma cocida, ni en forma de derivado (tipo miel de yacón). El yacón fue consumido adicional a su dieta.

Los pacientes fueron monitoreados semanalmente por 10 semanas y evaluados. Se les brindó consejería nutricional a sus familiares como parte del compromiso de la investigadora, tal como refiere el consentimiento informado.

Fueron excluidos del estudio los pacientes con diabetes mellitus, nefropatías crónicas, disfunción tiroidea, hepatopatías, afecciones gastrointestinales agudas, coronariopatías, abuso de fármacos o alcohol y HTA; pacientes con tratamiento hipolipemiante, por alterar los valores de los lípidos sanguíneos, por lo menos en los últimos 30 días; y mujeres que estén en etapa de menopausia y postmenopausia. 
Para el análisis estadístico se aplicó la prueba de hipótesis para diferencia de medias con muestras apareadas o aparejadas, para lo cual se calcularon las diferencias entre las medidas obtenidas al final del estudio y las obtenidas al inicio del estudio, a fin de ver si existía o no diferencia al aplicar el tratamiento. A la vez se calcularon promedios y desviaciones. El procesamiento estadístico de los datos se realizó por medio del programa SPSS 15.0 para Windows. Los gráficos fueron confeccionados en el programa Microsoft Excel 2003.

\section{RESULTADOS}

Tabla 4. Número de pacientes y Distribución porcentual según edad de los pacientes participantes en el estudio.

\begin{tabular}{|rcc|}
\hline Edad & Pacientes & $\%$ \\
\hline 35 a 49 & 5 & 5 \\
50 a 64 & 17 & 37.8 \\
65 a más & 23 & 51.1 \\
\hline TOTAL & 45 & 100.0 \\
\hline
\end{tabular}

Tabla 5. Pacientes dislipidémicos - grupo experimental y control según peso

\begin{tabular}{|c|c|c|c|c|c|c|c|c|}
\hline \multirow{3}{*}{ PESO } & \multicolumn{8}{|c|}{ CONTROL EXPERIMENTAL } \\
\hline & \multicolumn{2}{|c|}{ ANTES } & \multicolumn{2}{|c|}{ DESPUÉS } & \multicolumn{2}{|c|}{ ANTES } & \multicolumn{2}{|c|}{ DESPUÉS } \\
\hline & Pacientes & $\%$ & Pacientes & $\%$ & Pacientes & $\%$ & Pacientes & $\%$ \\
\hline$<60 \mathrm{Kg}$ & 4 & 25.0 & 3 & 18.7 & 9 & 31.0 & 10 & 34.5 \\
\hline 61 Kg. a 69 Kg. & 5 & 31.3 & 5 & 31.3 & 7 & 24.2 & 6 & 20.7 \\
\hline$>70 \mathrm{Kg}$ & 7 & 43.7 & 8 & 50.0 & 16 & 44.8 & 13 & 44.8 \\
\hline TOTAL & 16 & 100.0 & 16 & 100.0 & 29 & 100.0 & 29 & 100.0 \\
\hline
\end{tabular}

El $40 \%$ de la población estudiada estuvo integrada por mujeres y el $60 \%$ por varones. El $11.1 \%$ de los pacientes participantes tenía edades entre 35 y 49 años y el 51.1\% fueron mayores de 65 años (tabla 4). La mayoría de la población $(51.1 \%$ ) tenía una talla superior a $1.60 \mathrm{~m}$. El 43,7\% de los pacientes que pertenecieron al grupo control al inicio del estudio pesaban más de $70 \mathrm{Kg}$. y sólo el $25.0 \%$ tuvo un peso menor de $60 \mathrm{Kg}$. Al finalizar el estudio el 50\% de los pacientes que pertenecían al grupo control tuvo un peso superior a los $70 \mathrm{Kg}$. y sólo el $18.7 \%$ de los pacientes pesó menos de $60 \mathrm{Kg}$ (tabla 5). El 44.8\% de los pacientes que perteneció al grupo experimental al inicio del estudio pesaban más de $70 \mathrm{~kg}$ y sólo el $31.0 \%$ pesó menos de $60 \mathrm{~kg}$. Al finalizar el estudio un $44.8 \%$ de pacientes continuó con un peso superior a los $70 \mathrm{~kg}$. y el 34.5\% de los pacientes tuvo un peso menor de $60 \mathrm{~kg}$. Podemos notar en los pacientes dislipidémicos del grupo control y del grupo experimental que prevalece el sobrepeso, sobre todo antes de la intervención (43,7\% y 44.8\% grupo control y experimental respectivamente, de los pacientes pesaron más de $70 \mathrm{Kg}$.). Se puede indicar que el factor peso elevado en esta población, ha podido contribuir a la presencia de dislipidemia, sin descartar otros posibles factores como el estilo de vida.

Tabla 6. Pacientes dislipidémicos - Grupo control y grupo experimental según IMC

\begin{tabular}{|c|c|c|c|c|c|c|c|c|}
\hline \multirow{3}{*}{$\begin{array}{l}\text { Índice de Masa } \\
\text { Corporal }\end{array}$} & \multicolumn{8}{|c|}{ CONTROL EXPERIMENTAL } \\
\hline & \multicolumn{2}{|c|}{ ANTES } & \multicolumn{2}{|c|}{ DESPUÉS } & \multicolumn{2}{|c|}{ ANTES } & \multicolumn{2}{|c|}{ DESPUÉS } \\
\hline & Pacientes & $\%$ & Pacientes & $\%$ & Pacientes & $\%$ & Pacientes & $\%$ \\
\hline $18,5-24,9$ & 6 & 37.5 & 5 & 31.2 & 11 & 37.8 & 14 & 48.4 \\
\hline $25-29,9$ & 9 & 56.2 & 10 & 62.5 & 14 & 48.4 & 12 & 41.4 \\
\hline$>30$ & 1 & 6.3 & 1 & 6.3 & 4 & 13.8 & 3 & 10.2 \\
\hline TOTAL & 16 & 37.5 & 16 & 31.2 & 29 & 100.0 & 29 & 100.0 \\
\hline
\end{tabular}

El 56.2\% de los pacientes que perteneció al grupo control al inicio del estudio, tuvieron un índice de masa corporal entre 25 - 29.9 (sobrepeso) el 37.5\% tuvo entre 18.5 - 24.9 (normal). Al finalizar el estudio, el 62.5\% de los pacientes que pertenecía al grupo control tuvo un índice de masa corporal entre 25 - 29.9 y el $31.2 \%$ un índice entre 18.5 y 24.9. El $48.4 \%$ de los pacientes que pertenecó al grupo experimental al inicio del estudio tuvo un índice de masa corporal entre 25 - 29.9 (sobrepeso), el $37.8 \%$ tuvo entre 18.5 - 24.9 (normal) y el $13.8 \%$ de los pacientes tuvo entre más de 30 (Obesidad). Al finalizar el estudio el 48.4\% de los pacientes tuvieron un índice de masa corporal entre 18.5 - 24.9 (normal) y el 41.4\% de los pacientes un índice entre 25 - 29.9 (sobrepeso) (tabla 6). 
Tabla 7. Pacientes dislipidémicos - Grupo control y grupo experimental, según riesgo coronario.

\begin{tabular}{|c|c|c|c|c|c|c|c|c|c|}
\hline \multirow{3}{*}{ SEXO } & \multirow{3}{*}{$\begin{array}{c}\text { R.C. } \\
\text { según } \\
\text { índice } \\
\text { Castelli }\end{array}$} & \multicolumn{8}{|c|}{ CONTROL EXPERIMENTAL } \\
\hline & & \multicolumn{2}{|c|}{ ANTES } & \multicolumn{2}{|c|}{ DESPUÉS } & \multicolumn{2}{|c|}{ ANTES } & \multicolumn{2}{|c|}{ DESPUÉS } \\
\hline & & Pacientes & $\%$ & Pacientes & $\%$ & Pacientes & $\%$ & Pacientes & $\%$ \\
\hline \multirow{3}{*}{ Mujeres } & $3,4-4,5$ & 2 & 33.3 & 0 & 0.0 & 0 & 0.0 & 3 & 25.0 \\
\hline & $4,5-7,1$ & 4 & 66.7 & 5 & 83.3 & 11 & 91.6 & 8 & 66.6 \\
\hline & $7,2-11$ & 0 & 0.0 & 1 & 16.7 & 1 & 8.4 & 1 & 8.4 \\
\hline \multicolumn{2}{|c|}{ TOTAL } & 6 & 100.0 & 6 & 100.0 & 12 & 100.0 & 12 & 100.0 \\
\hline \multirow{3}{*}{ Hombre } & $<3.5$ & 0 & 0 & 0 & 0 & 0 & 0.0 & 2 & 11.8 \\
\hline & $3,5-5,0$ & 1 & 10.0 & 0 & 0.0 & 2 & 11.8 & 7 & 41.2 \\
\hline & $5,1-9,6$ & 9 & 90.0 & 10 & 100.0 & 15 & 88.2 & 8 & 47.0 \\
\hline \multicolumn{2}{|c|}{ TOTAL } & 10 & 100.0 & 10 & 100.0 & 17 & 100.0 & 17 & 100.0 \\
\hline
\end{tabular}

Al inicio del estudio, el 66.7\% de las mujeres del grupo control tuvieron riesgo cardiaco (tabla 7) entre 4.5 - 7.1, después del estudio un 83.3\% tuvo el mismo riesgo cardiaco. En cuanto a los varones después del estudio el 100\% de ellos tuvo riesgo cardíaco entre 5,1 - 9.6. El riesgo cardíaco promedio al inicio del estudio fue de 5.4 y al finalizar el estudio fue de 6.3. Este aumento es estadísticamente significativa $(\mathrm{p}<0.05)$, por lo tanto el riesgo cardíaco aumentó al finalizar el estudio en los pacientes que no recibieron tratamiento con yacón. Al inicio del estudio el $91.6 \%$ de las mujeres del grupo experimental tuvieron riesgo cardiaco entre $4.5-7.1$, después del estudio sólo el $66.6 \%$ tuvo el mismo riesgo cardíaco. A la vez un 8.45 de las mujeres tienen riesgo cardiaco entre 7,2 - 11 antes y después del estudio. En cuanto a los varones después del estudio sólo un 47.0\% de ellos tuvo riesgo cardíaco entre 5,1 - 9.6. El riesgo cardiaco promedio en pacientes del grupo experimental, al inicio del estudio fue de 6.0 y al finalizar el estudio fue de 5.0. Esta disminución es estadísticamente significativa $(\mathrm{p}<0.05)$, por lo tanto, el riesgo cardíaco disminuyó al finalizar el estudio.

Tabla 8. Pacientes dislipidémicos - Grupo control y grupo experimental, según niveles de VLDL

\begin{tabular}{|c|c|c|c|c|c|c|c|c|}
\hline \multirow{3}{*}{ VLDLmg/dl } & \multicolumn{8}{|c|}{ CONTROL EXPERIMENTAL } \\
\hline & \multicolumn{2}{|c|}{ ANTES } & \multicolumn{2}{|c|}{ DESPUÉS } & \multicolumn{2}{|c|}{ ANTES } & \multicolumn{2}{|c|}{ DESPUÉS } \\
\hline & Pacientes & $\%$ & Pacientes & $\%$ & Pacientes & $\%$ & Pacientes & $\%$ \\
\hline $5.0-40.0$ & 13 & 81.3 & 7 & 43.8 & 16 & 55.2 & 23 & 79.3 \\
\hline$>40.0$ & 3 & 18.7 & 9 & 56.2 & 13 & 44.8 & 6 & 20.7 \\
\hline TOTAL & 16 & 100.0 & 16 & 100.0 & 29 & 100.0 & 29 & 100.0 \\
\hline
\end{tabular}

Al inicio del estudio, el $81.3 \%$ de los pacientes que perteneció al grupo control tuvieron rangos de VLDL (Tabla No 8) entre 5 - 40.0 (normal) y el $18.7 \%$ de los pacientes más de 40.0 . Al finalizar el estudio el 56.2\% de los pacientes tuvo niveles de VLDL mayor a 40.0 y un $43.8 \%$ de los pacientes entre 5 - 40.0 de lipoproteínas VLDL. El nivel promedio de VLDL antes del estudio fue de 30.9 y después del estudio fue 43.9. Este aumento es estadísticamente significativa $(\mathrm{p}<0.05)$, por lo tanto los niveles de VLDL aumentaron al finalizar el estudio. En los pacientes que pertenecieron al grupo experimental, el 55.2\% presentó niveles de VLDL entre 5 - 40 (normal). Después de aplicar el tratamiento el 79.3\% de los pacientes de este grupo tuvo niveles VLDL entre 5-40 (normal). El nivel promedio de VLDL al inicio del programa, en los pacientes que pertenecen al grupo experimental, fue de 38.1 y después de aplicar el tratamiento el promedio de VLDL fue de 30.2. Esta disminución es significativa estadísticamente $(p<0.05)$, por lo tanto los niveles de VLDL disminuyeron al finalizar el estudio.

Tabla 9. Pacientes dislipidémicos - Grupo control y grupo experimental según niveles de LDL

\begin{tabular}{|c|c|c|c|c|c|c|c|c|}
\hline \multirow{3}{*}{ LDL mg/dl } & \multicolumn{8}{|c|}{ CONTROL EXPERIMENTAL } \\
\hline & \multicolumn{2}{|c|}{ ANTES } & \multicolumn{2}{|c|}{ DESPUÉS } & \multicolumn{2}{|c|}{ ANTES } & \multicolumn{2}{|c|}{ DESPUÉS } \\
\hline & Pacientes & $\%$ & Pacientes & $\%$ & Pacientes & $\%$ & Pacientes & $\%$ \\
\hline$<129.0$ & 0 & 0.0 & 0 & 0.0 & 3 & 10.3 & 8 & 27.6 \\
\hline $130.0-159.0$ & 11 & 68.8 & 5 & 31.2 & 6 & 20.7 & 10 & 34.5 \\
\hline$>160.0$ & 5 & 31.2 & 11 & 68.8 & 20 & 69.0 & 11 & 37.9 \\
\hline TOTAL & 16 & 100.0 & 16 & 100.0 & 29 & 100.0 & 29 & 100.0 \\
\hline
\end{tabular}


En cuanto a los niveles de LDL (tabla No 9), un 68.8\% de los pacientes del grupo control al inicio del estudio tuvo entre 130 - 159.0 (límite), un $31.2 \%$ de los pacientes presentaron niveles de LDL elevados. Al finalizar el estudio, el $68.8 \%$ de los pacientes del grupo control presentó niveles de LDL mayor a 160.0 (elevado). Al inicio del estudio, en promedio el nivel de LDL fue de 158.8 y al finalizar el estudio fue de 169.3. Este aumento es estadísticamente significativa $(\mathrm{p}<0.05)$, por lo tanto los niveles de LDL aumentaron al finalizar el estudio. Al inicio del estudio, el $69.0 \%$ de los pacientes que pertenecieron al grupo experimental tuvo un niveles de LDL mayor a 160.0 (elevado), después de aplicar el tratamiento solo un $37.9 \%$ de los pacientes presentó niveles de LDL mayor a 160.0 (elevado) y un $27.6 \%$ de los pacientes presentó niveles de LDL menor a 129.9 (deseables). En los pacientes del grupo experimental, el nivel promedio de LDL al inicio del estudio fue de 184.1 y al finalizar el estudio el promedio de LDL fue de 153.6. Esta disminución es significativa estadísticamente $(\mathrm{p}<0.05)$, por lo tanto los niveles de LDL disminuyeron al finalizar el estudio.

Tabla 10. Pacientes dislipidémicos - Grupo control y grupo experimental según niveles de HDL

\begin{tabular}{|c|c|c|c|c|c|c|c|c|c|}
\hline \multirow{3}{*}{ SEXO } & \multirow{3}{*}{$\begin{array}{l}\text { HDL } \\
\mathrm{mg} / \mathrm{dl}\end{array}$} & \multicolumn{8}{|c|}{ CONTROL EXPERIMENTAL } \\
\hline & & \multicolumn{2}{|c|}{ ANTES } & \multicolumn{2}{|c|}{ DESPUÉS } & \multicolumn{2}{|c|}{ ANTES } & \multicolumn{2}{|c|}{ DESPUÉS } \\
\hline & & Pacientes & $\%$ & Pacientes & $\%$ & Pacientes & $\%$ & Pacientes & $\%$ \\
\hline \multirow{3}{*}{ Mujeres } & $>45$ & 2 & 33.3 & 1 & 16.7 & 8 & 66.7 & 8 & 66.7 \\
\hline & $44-34$ & 4 & 66.7 & 4 & 66.7 & 4 & 33.3 & 4 & 33.3 \\
\hline & $<35$ & 0 & 0.0 & 1 & 16.7 & 0 & 0.0 & 0 & 0.0 \\
\hline \multicolumn{2}{|c|}{ TOTAL } & 6 & 100.0 & 6 & 100.0 & 12 & 100.0 & 12 & 100.0 \\
\hline \multirow{3}{*}{\multicolumn{2}{|c|}{ Hombre }} & 7 & 70.0 & 4 & 40.0 & 8 & 47.0 & 10 & 58.9 \\
\hline & & 2 & 20.0 & 4 & 40.0 & 4 & 23.5 & 5 & 29.4 \\
\hline & & 1 & 10.0 & 2 & 20.0 & 5 & 29.4 & 2 & 11.7 \\
\hline \multicolumn{2}{|c|}{ TOTAL } & 10 & 100.0 & 10 & 100.0 & 17 & 100.0 & 17 & 100.0 \\
\hline
\end{tabular}

La clasificación de las lipoproteínas HDL (tabla 10) son diferentes de acuerdo al sexo. En el grupo control al inicio del estudio, un $66.7 \%$ de las mujeres presentó niveles de HDL entre $44-34$, de los hombres el $70 \%$ presentó niveles deseables de HDL. Sin embargo, al finalizar el estudio un $16.7 \%$ de las pacientes mujeres presentaron niveles elevados de HDL (menor a 35) y de los hombres un $20 \%$ presentó niveles elevados de lipoproteínas HDL. Al inicio del estudio el nivel promedio de HDL fue de 43.0 y al finalizar el estudio fue de 40.9. Esta disminución estadísticamente no significativa ( $>0.05$ ), por lo tanto los niveles de HDL no disminuyeron al finalizar el estudio. Al inicio del estudio el $66.7 \%$ de las pacientes mujeres que perteneció al grupo experimental presentó niveles de HDL mayor a 45 (deseable), lo cual se mantuvo hasta el final del estudio. En el grupo de los varones, el $47 \%$ al inicio del estudio mostró niveles de HDL menores a 40 y después del estudio un $58.9 \%$ presentó similares niveles. Al inicio del estudio, el nivel promedio de HDL fue de 45.4 y al finalizar el estudio fue de 46.2. Este aumento es estadísticamente no significativa ( $>0.05$ ), por lo tanto los niveles de HDL no aumentaron al finalizar el estudio.

Tabla 11. Pacientes dislipidémicos - Grupo control y grupo experimental según niveles de colesterol

\begin{tabular}{|c|c|c|c|c|c|c|c|c|}
\hline \multirow{3}{*}{$\begin{array}{l}\text { COLESTEROL } \\
\text { LDL mg/dl }\end{array}$} & \multicolumn{8}{|c|}{ CONTROL EXPERIMENTAL } \\
\hline & \multicolumn{2}{|c|}{ ANTES } & \multicolumn{2}{|c|}{ DESPUÉS } & \multicolumn{2}{|c|}{ ANTES } & \multicolumn{2}{|c|}{ DESPUÉS } \\
\hline & Pacientes & $\%$ & Pacientes & $\%$ & Pacientes & $\%$ & Pacientes & $\%$ \\
\hline$<199.0$ & 0 & 0.0 & 1 & 6.3 & 1 & 3.4 & 7 & 24.2 \\
\hline $200-239.0$ & 10 & 62.5 & 5 & 31.2 & 5 & 17.2 & 13 & 44.8 \\
\hline$>240.0$ & 6 & 37.5 & 10 & 62.5 & 23 & 79.3 & 9 & 31.0 \\
\hline TOTAL & 16 & 100.0 & 16 & 100.0 & 29 & 100.0 & 29 & 100.0 \\
\hline
\end{tabular}

En el grupo control un $62.5 \%$ de los pacientes, al inicio del estudio presentó niveles de colesterol límites (tabla 11) y un $37.5 \%$ de los pacientes niveles elevados. Al finalizar el estudio el $62.5 \%$ de los pacientes presentó niveles elevados de colesterol. Al inicio del estudio los pacientes que pertenecieron al grupo control tuvieron en promedio 232.6 de colesterol, al finalizar el estudio aumentaron a 254.0. Este aumento es estadísticamente significativa $(\mathrm{p}<0.05)$, por lo tanto los niveles de colesterol aumentaron al finalizar el estudio. En el grupo experimental, un $79.3 \%$ de los pacientes, al inicio del estudio presentó niveles de colesterol elevados; sin embargo al finalizar el estudio sólo el $31.0 \%$ permaneció en el mismo nivel, un $24.2 \%$ de los pacientes se encontró niveles de colesterol deseables. 
Al inicio del estudio los pacientes que pertenecieron al grupo experimental tuvieron en promedio $267.7 \mathrm{mg} / \mathrm{dl} \mathrm{de}$ colesterol, y al finalizar el estudio disminuyeron a $230.1 \mathrm{mg} / \mathrm{dl}$. Esta disminución es estadísticamente significativa $(\mathrm{p}<0.05)$, por lo tanto los niveles de colesterol disminuyeron con la aplicación del tratamiento.

Tabla 12. Pacientes dislipidémicos - Grupo control y grupo experimental según niveles de triglicéridos.

\begin{tabular}{|c|c|c|c|c|c|c|c|c|}
\hline \multirow{2}{*}{$\begin{array}{l}\text { TRIGLICERIDOS } \\
\text { LDL mg/dl }\end{array}$} & \multicolumn{8}{|c|}{ CONTROL EXPERIMENTAL } \\
\hline & \multicolumn{2}{|c|}{ ANTES } & \multicolumn{2}{|c|}{ DESPUÉS } & \multicolumn{2}{|c|}{ ANTES } & \multicolumn{2}{|c|}{ DESPUÉS } \\
\hline$<199.0$ & 13 & 81.2 & 7 & 43.8 & 17 & 58.6 & 23 & 79.3 \\
\hline $200.0-400.0$ & 3 & 18.8 & 9 & 56.2 & 12 & 41.4 & 6 & 20.7 \\
\hline
\end{tabular}

En el grupo control un $81.2 \%$ de los pacientes, al inicio del estudio presentó niveles de triglicéridos deseables (Tabla 12) (menor a 199.0) y un $18.8 \%$ de los pacientes niveles límites. Al finalizar el estudio el $56.2 \%$ de los pacientes presentó niveles límites de triglicéridos. Al inicio del estudio los pacientes que pertenecieron al grupo control tuvieron en promedio $149.3 \mathrm{mg} / \mathrm{dl}$ de triglicéridos, al finalizar el estudio los valores aumentaron a $217.1 \mathrm{mg} / \mathrm{dl}$ en promedio. Este aumento es estadísticamente significativa $(\mathrm{p}<0.05)$, por lo tanto los niveles de triglicéridos aumentaron al finalizar el estudio. En el grupo experimental un 58.6\% de los pacientes, al inicio del estudio presentó niveles de triglicéridos deseables, sin embargo al finalizar el estudio aumentó la proporción de pacientes en este nivel (79.3\%), aún un $20.7 \%$ de los pacientes al finalizar el estudio se encuentra en niveles limites de triglicéridos. Al inicio del estudio los pacientes que pertenecieron al grupo experimental tuvieron en promedio 185.2 de triglicéridos, al finalizar el estudio el promedio disminuyó a $149.4 \mathrm{mg} / \mathrm{dl}$. Esta disminución es estadísticamente significativa $(\mathrm{p}<0.05)$, por lo tanto los niveles de triglicéridos disminuyeron con la aplicación del tratamiento.

\section{DISCUSIÓN Y CONCLUSIONES}

Según los resultados obtenidos, el consumo de yacón redujo significativamente $(\mathrm{p}<0.05)$ los niveles promedio de VLDL (de 38.1 a $30.2 \mathrm{mg} / \mathrm{dl}$ ), LDL (de 184.1 a $153.6 \mathrm{mg} / \mathrm{dl}$ ), colesterol total (de 267 a 230.1 mg/dl) y triglicéridos (de $185.2149 .4 \mathrm{mg} / \mathrm{dl}$ ), sin modificar los niveles de HDL colesterol los cuales se mantuvieron similares hasta el final del estudio. En conjunto, el impacto sobre las variables mencionadas disminuyó significativamente $(\mathrm{p}<0.05)$ el riesgo cardíaco (de 6.0 a 5.0).

Otros estudios han mostrados efectos similares. Enzio Foy Valencia ${ }^{(7)}$, aplicó el Smallanthus sonchifolius (yacón) en el tratamiento de la hiperlipoproteinemia e hipercolesterolemia inducidas en ratas albinas. Trabajó con dos bloques, uno para el control y otro para la aplicación experimental. Al bloque control se le administró una dieta libre de colesterol, la cual estuvo conformada por insumos de origen vegetal. Al bloque experimental se le indujo una colesterolemia, dándole una dieta con hígado de res la primera semana y yema de huevo la segunda semana.

Posteriormente, al bloque experimental inducido a la colesterolemia se le administró una dieta suplementada con “yacón”. Tanto al bloque control como al experi- mental se le efectuó un análisis bioquímico del suero sanguíneo con el objetivo de determinar su perfil lipídico en condiciones basales. Con el bloque experimental se efectuó un segundo análisis bioquímico del suero, luego de haberse aplicado la dieta rica en grasa para verificar si se indujo a la colesterolemia. Al cabo de haberse administrado la dieta suplementada con "yacón”, se realizó un tercer análisis bioquímico del suero sanguíneo para comprobar el efecto sobre los niveles de lípidos del producto investigado. Se concluyó que el nivel sanguíneo de colesterol total en condiciones basales para las ratas albinas fue de $86,66 \mathrm{mg} / \mathrm{dl}$; éste fue elevado con la dieta hasta $188,88 \mathrm{mg} / \mathrm{dl}$; y luego de administrarle el yacón se redujo hasta $85,33 \mathrm{mg} / \mathrm{dl}$. Lo mismo sucedió con las lipoproteínas de baja densidad (LDL) las cuales tuvieron un valor basal de 36,05 $\mathrm{mg} / \mathrm{dl}$; con la dieta grasa se elevó hasta $123,95 \mathrm{mg} / \mathrm{dl}$; para que finalmente con la dieta con yacón disminuyera hasta valores de $62,98 \mathrm{mg} / \mathrm{dl}$. En lo que corresponde a los triglicéridos, éstos tuvieron un valor sanguíneo basal de 166,66 mg/dl; con la dieta grasa se elevaron hasta $181,81 \mathrm{mg} / \mathrm{d}$; para posteriormente con la dieta con yacón se redujo hasta $36,36 \mathrm{mg} / \mathrm{dl}^{(7)}$.

Christine Williams, de la Universidad de Reading del Reino Unido, reportó en su trabajo de investigación que una dieta suplementada con $10 \mathrm{~g} /$ día de inulina durante 8 semanas, puede hacer decrecer significativamente el 
nivel de triglicéridos en la sangre ${ }^{(15)}$. Balcázar (2003)

(16) en la investigación realizada con respecto al efecto de la administración oral de inulina sobre el perfil de lípidos y la sensibilidad a la insulina en individuos con obesidad y dislipidemia, a quienes administró $7 \mathrm{~g} /$ día de inulina durante 4 semanas, logró una disminución significativa de las VLDL, siendo al inicio de $45.9 \mathrm{mg} /$ dl y después de la ingesta de inulina fue de $31.6 \mathrm{mg} / \mathrm{dl}$, $(p=0.046)^{(17)}$, lo cual es congruente con nuestros resultados. Contreras (2008), refiere en el estudio denominado, Factores de riesgo cardiovascular en población adulta, que las poderosas influencias socioculturales de la familia, el trabajo y la comunidad afectan el comportamiento individual y por tanto a los niveles de factores de riesgo y los hispanos tienen mayor prevalencia de factores de riesgo como diabetes, obesidad, anormalidades de los lípidos y sedentarismo. Asimismo, refiere que el panel de expertos en detección, evaluación y tratamiento de la hipercolesterolemia en adultos, (Tercer Reporte Nacional del Programa de Educación en Colesterol) enfatiza que los efectos cuantitativos de los cambios dietéticos sobre las LDL por medio de la dieta alta en colesterol incrementa las LDL, observándose que en promedio un incremento de $100 \mathrm{mg}$ de colesterol al día en la dieta resulta en el incremento de 2 a 3 $\mathrm{mg} / \mathrm{dl}$ del colesterol sérico, del cual $70 \%$ representa la fracción LDL.

Por lo que recomienda planes de alimentación con el incremento del consumo de fibra soluble (10 a 25g/día). Esta sola estrategia disminuye el colesterol LDL de 11 a 15\%.(16). Así mismo, el Dr. Philip Barter (2007)(18), en el informe que aparece en la edición del 27 de septiembre del New England Journal of Medicine refiere que la incidencia de ataque cardíaco, accidente cerebrovascular y otros problemas cardíacos fue 40 por ciento menor en la quinta parte de los participantes en un importante ensayo que tenían los niveles más altos de colesterol HDL, independientemente de sus niveles de colesterol LDL, "El mensaje fundamental e importante del artículo es que si se lleva el HDL suficientemente alto, el LDL no importa”, apuntó el autor del estudio, el Dr. Philip Barter, director del Instituto de investigación cardiaca de Sídney, Australia.
Ahora bien, el yacón no sólo ha mostrado tener efectos hipolipemiantes, sino que también posee efectos antihiperglicemiantes. Mayta, P. y Col. estudiaron la reducción de la respuesta glicémica posprandial postingesta de raíz fresca de yacón en sujetos sanos. En este estudio clínico experimental no ciego, participaron 6 sujetos sanos los cuales fueron sometidos primero a un test de tolerancia oral a la glucosa (TTOG -control), y luego un test similar añadiendo $300 \mathrm{~g}$ de raíz fresca de yacón vía oral (TTOG-yacón). Se midieron los niveles de glucosa a los 0, 30, 60, 90 y 120 minutos. Como resultado, se evidenció una reducción de $79.8 \%(\mathrm{p}=0.001)$ de la respuesta glicémica posprandial con el TTOG yacón, además de un mínimo pico posprandial de glucosa a los 30 minutos $(p=0.0016)$. La máxima diferencia entre los niveles de glicemia en ambos grupos de estudio se da a los 60 minutos $(\mathrm{p}=$ 0.0021). El trabajo concluye que el consumo agudo de la raíz fresca de yacón reduce la respuesta glicémica posprandial en sujetos sanos ${ }^{(19)}$. Aybar, M., Sánchez, R. y Col. estudiaron el efecto de la administración durante 30 días de té de yacón a ratas con diabetes inducida por estreptozotocina (STZ). Los resultados mostraron efecto reductor de los niveles de glucosa, las ratas con diabetes tuvieron 5,81 g/L de glucosa en plasma mientras que las ratas diabéticas tratadas con yacón redujeron su glucosa a 4,20 g/L. En la capacidad de aumentar los niveles de insulina en sangre, las ratas diabéticas presentaron $1,0 \mathrm{uU} / \mathrm{ml}$ de insulina y las ratas diabéticas tratadas con yacón incrementaron su insulina a 3,3 uU/ $\mathrm{ml}$. Y en la capacidad de mantener filtrante los riñones, cuya falla se evidencia por aumento en la eliminación de creatinina y de albúmina, las ratas diabéticas presentaron valores de $1,26 \mathrm{ml} / \mathrm{min}$ de eliminación de creatinina y las ratas tratadas con yacón redujeron su excreción a $0,91 \mathrm{ml} / \mathrm{min}^{(20)}$.

En conclusión, el consumo diario de yacón ha mostrado reducir significativamente $(\mathrm{p}<0.05)$ los niveles promedio de VLDL, LDL, colesterol total y triglicéridos sin modificar los niveles de HDL colesterol. 


\section{REFERENCIAS BIBLIOGRÁFICAS}

1. Balcázar-Muñoz, Blanca R y Col. Efecto de la administración oral de inulina sobre el perfil de lipidos y la sensibilidad a la insulina en individuos con obesidad y dislipidemia. Rev. Méd. Chile, vol.131, no.6; jun. 2003. p.597-604. ISSN 0034-9887.

2. Ccama, Faustino. Proyecto R6-004: Evaluación, mejoramiento y difusión de los sistemas de conservación y transformación de raíces y tubérculos andinos; 1996.

3. Cisneros-Zevallos, L. Characterization and Evaluation of Fructooligosaccharides on Yacon Roots (Smallanthus sonchifolius Poepp. \& Endl.) During Storage. Department of Horticulture, Texas; 2002.

4. Collazos, C.. La composición de los alimentos peruanos. Quinta Edición, Ministerio de Salud, INS, Lima, Perú; 1975.

5. Enzio Foy Valencia. Smallanthus Sonchifolius (llacón o yacón) en el tratamiento de hiperlipoproteinemias e hipercolesterolemia inducidas en ratas albinas. Rev. Facultad Medicina Humana; 2005; 5 (1.): 27-31.

6. Gotto AM, Assmann NG, Carmena R, Davignon J, Fernández-Cruz A, Paoletti R. The ILIB Spectial consideration in the elderly. En: the International Lipid Information Bureau eds. Handbook for clinical practice. Blood lipids and coronary heart disease. 1995. p.155.

7. Graefe S, Hermann M, Manrique I, Golombek S \& A Bürkert. Effects of post-harvest treatments on the carbohydrate composition of yacón roots in the Peruvian Andes. Field Crops Research 86; 2004. 157-165

8. Grau, A. y REA J.. Yacón, Smallanthus sonchifolius (Poepp. \& Endl.) H. Robinson. En: Hermann, M. \& Heller J. (Editors), Andean roots and tubers: Ahipa, arracacha, maca and yacón, IPK and IPGRI, Rome; 1997. pp. 200-242.

9. http://medicinalternativa.blogspot.com/2006/01/ el-yacon-para-los diabeticos.html.

10. Lopes-Virella MF. Stone P. Cholesterol determination in high-density lipoproteins separated by three different methods. Clin Chem; 1997. 23:882-884.
11. Mataix Verdú. Nutrición y Alimentación Humana. Situaciones fisiológicas y patológicas. ERGON. Madrid; 2002.

12. Ministerio de Salud. Encuesta nacional de indicadores nutricionales, bioquímicos, socioeconómicos y culturales relacionados con las enfermedades crónico degenerativas. Lima Perú; 2006.

13. NHANES, Prevención de la enfermedades cardiovasculares en mujeres: Una propuesta para América Latina y el Caribe; 2000.

14. Pedreschi, D. Campos y Col. "Fermentación de Fructooligosacáridos de Yacón por L. bifidum acidophilus, L. bifidum brevis, L. bifidum gasseri, L. bifidum plantarum y bifidobacteria. Annual meeting and food Expo Anaheim”, California. USA. 2002.

15. Segundo Seclén, Julio Leey y Col. Prevención de Diabetes Mellitus, Hipertensión Arterial, Hipercolesterolemia y Obesidad, como Factores de Riesgo Coronario y Cerebrovascular en Población Adulta de la Costa, Sierra y Selva del Perú. Revista Peruana de Cardiología; 2000.

16. Serra, César; Sala P. José y Balestrini C. Epidemiología. Instituto Modelo de Cardiología. Privado SRL. Córdoba. Municipalidad de Carlos Paz. Córdoba, Argentina; 2004.

17. Castelli WP, Garrison RJ, Wilson PW, Abbott RD, Kalousdian S, Kannel WB. Incidence of coronary heart disease and lipoprotein cholesterol level: The Framingham Study. JAMA; 1986; 256: 2835-8.

18. Barter, Philip. M.D., director, Heart Research Institute, Sydney, Australia; Vera Bittner, professor, medicine. University of Alabama at Birmingham; Sept. 27; 2007, New England Journal of Medicine.

19. Mayta, Percy, PAYANO, Jyp, PELAEZ, Joel et al. Reducción de la respuesta glicémica posprandial post-ingesta de raíz fresca de yacón en sujetos sanos. CIMEL; 2004, vol.9, no.1, p.7-11. ISSN 16808398.

20. Aybar, M. /Graú, A. / Sanchez Riera, A. \& Sanchez, S. Hypoglycemic effect of the water extract of Smallanthus sonchifolius (Yacón) leaves in normal and diabetic rats. Journal of Ethnopharmacology; 2001; 74: 125-132. 